\title{
0933 CHILD AND ADOLESCENT SEXUAL ABUSE AND
} VIOLENCE IN INDIA: A REVIEW

S Malhotra* Correspondence: Indian Institute of Public Health, Public Health Foundation of India, B-3/84 A, Mig DDA Flats, Lawrence Road, Keshav Puram, New Delhi 110035, India

\subsection{6/ip.2010.029215.933}

In recent years, there has been an increasing attention to sexual coercive experiences of young people in developing countries, including India. Research done in this newer area is generating new evidences that call for more specific interventions. It is imperative that the findings coming from qualitative and quantitative research should be assimilated to present the perspectives in this context for a diverse group encompassing married and unmarried young people aged. Thus a review was undertaken to determine prevalence of different non-consensual sexual experiences and the related correlates found out through multivariate analysis. A thorough search was done using key search engines, manual search of grey literature, websites, cross references of articles searched in English language. Sexual abuse and violence is observed in both Indian boys and girls in their early years of life. Both community and facility based studies have shown traumatic and painful experiences of young people. $13 \%-32 \%$ boys and $12 \%-42 \%$ girls have reported unwanted touching, $4 \%-15 \%$ boys and $3 \%$ of girls reported forced physical relationship. Evidence is also available to suggest vulnerability for street children for facing such adverse experiences. Older children, neighbours and strangers are most common perpetrators reported. Having a friend of opposite gender, poor academic performance, poor mental and physical health, greater substance abuse, poorer parental relationships are associated factors for undergoing coercive behaviour. Urban rural differences also exist. The findings underscore the need to develop programs that enable children and adolescents to prevent and cope with non-consensual sexual experiences. 